\title{
Crystal structure of $(P)-4,4^{\prime}$-di-tert-butyl-2,2',3,3'-tetramethoxy- 6,6'-dimethylbiphenyl, $\left\{\mathrm{C}_{6} \mathrm{H}\left(\mathrm{OCH}_{3}\right)_{2}\left(\mathrm{CH}_{3}\right)\left[\mathrm{C}\left(\mathrm{CH}_{3}\right)_{3}\right]\right\}_{2}$
}

\author{
K. Peters* ${ }^{*}$, E.-M. Peters', T. Pabst ${ }^{\mathrm{II}}$ and G. Bringmann ${ }^{\mathrm{II}}$ \\ I Max-Planck-Institut für Festkörperforschung, Heisenbergstraße 1, D-70506 Stuttgart, Germany \\ II Universität Würzburg, Institut für Organische Chemie, Am Hubland, D-97074 Würzburg, Germany
}

Received December 9, 1999, CCDC-No. 1267/356

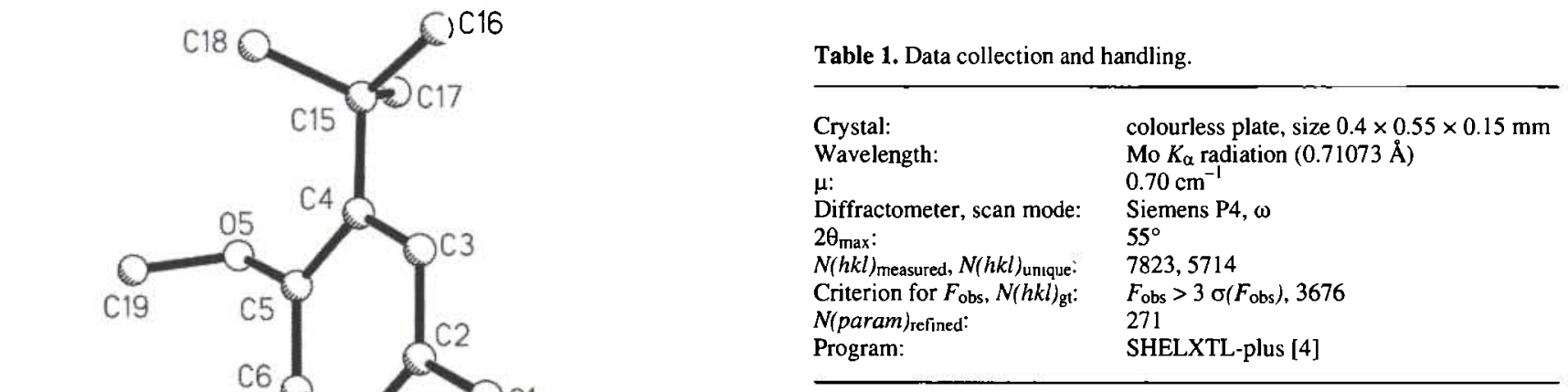

Table 2. Atomic coordinates and displacement parameters (in $\AA^{2}$ ).

\begin{tabular}{|c|c|c|c|c|c|}
\hline Atom & Site & $x$ & $y$ & $z$ & $U_{i s \mathrm{o}}$ \\
\hline$H(1 A)$ & $4 a$ & $1.0270(6)$ & $0.3512(3)$ & $0.1820(1)$ & 0.08 \\
\hline$H(1 B)$ & $4 a$ & $1.0448(6)$ & $0.4053(3)$ & $0.1272(1)$ & 0.08 \\
\hline $\mathrm{H}(1 \mathrm{C})$ & $4 a$ & $1.1939(6)$ & $0.3517(3)$ & $0.1546(1)$ & 0.08 \\
\hline$H(3)$ & $4 a$ & $1.1823(4)$ & $0.2537(3)$ & $0.0615(1)$ & 0.08 \\
\hline $\mathrm{H}(12)$ & $4 a$ & $0.9302(4)$ & $0.1432(3)$ & $0.3247(1)$ & 0.08 \\
\hline $\mathrm{H}(14 \mathrm{~A})$ & $4 a$ & $1.1503(4)$ & $0.0864(3)$ & $0.2100(1)$ & 0.08 \\
\hline $\mathrm{H}(14 \mathrm{~B})$ & $4 a$ & $1.1140(4)$ & $0.0213(3)$ & $0.2613(1)$ & 0.08 \\
\hline $\mathrm{H}(14 \mathrm{C})$ & $4 a$ & $1.1893(4)$ & $0.1434(3)$ & $0.2630(1)$ & 0.08 \\
\hline $\mathrm{H}(16 \mathrm{~A})$ & $4 a$ & $1.3394(6)$ & $0.1806(4)$ & $0.0030(1)$ & 0.08 \\
\hline $\mathrm{H}(16 \mathrm{~B})$ & $4 a$ & $1.1905(6)$ & $0.2407(4)$ & $-0.0216(1)$ & 0.08 \\
\hline $\mathrm{H}(16 \mathrm{C})$ & $4 a$ & $1.2926(6)$ & $0.1552(4)$ & $-0.0542(1)$ & 0.08 \\
\hline $\mathrm{H}(17 \mathrm{~A})$ & $4 a$ & $1.3185(7)$ & $-0.0297(4)$ & $0.0233(1)$ & 0.08 \\
\hline $\mathrm{H}(17 \mathrm{~B})$ & $4 a$ & $1.2810(7)$ & $-0.0490(4)$ & $-0.0351(1)$ & 0.08 \\
\hline $\mathrm{H}(17 \mathrm{C})$ & $4 a$ & $1.1634(7)$ & $-0.0953(4)$ & $0.0069(1)$ & 0.08 \\
\hline $\mathrm{H}(18 \mathrm{~A})$ & $4 a$ & $0.9358(5)$ & $0.0075(4)$ & $-0.0347(1)$ & 0.08 \\
\hline $\mathrm{H}(18 \mathrm{~B})$ & $4 a$ & $1.0536(5)$ & $0.0534(4)$ & $-0.0768(1)$ & 0.08 \\
\hline $\mathrm{H}(18 \mathrm{C})$ & $4 a$ & $0.9515(5)$ & $0.1389(4)$ & $-0.0442(1)$ & 0.08 \\
\hline $\mathrm{H}(19 \mathrm{~A})$ & $4 a$ & $0.7287(6)$ & $-0.1542(5)$ & $0.0245(2)$ & 0.08 \\
\hline $\mathrm{H}(19 \mathrm{~B})$ & $4 a$ & $0.7213(6)$ & $-0.0217(5)$ & $0.0176(2)$ & 0.08 \\
\hline $\mathrm{H}(19 \mathrm{C})$ & $4 a$ & $0.6883(6)$ & $-0.0762(5)$ & $0.0716(2)$ & 0.08 \\
\hline $\mathrm{H}(20 \mathrm{~A})$ & $4 a$ & $0.7903(6)$ & $-0.1674(4)$ & $0.1844(2)$ & 0.08 \\
\hline $\mathrm{H}(20 \mathrm{~B})$ & $4 a$ & $0.9462(6)$ & $-0.0969(4)$ & $0.1947(2)$ & 0.08 \\
\hline $\mathrm{H}(20 \mathrm{C})$ & $4 a$ & $0.9237(6)$ & $-0.1604(4)$ & $0.1422(2)$ & 0.08 \\
\hline $\mathrm{H}(22 \mathrm{~A})$ & $4 a$ & $0.8565(5)$ & $0.2349(3)$ & $0.3950(1)$ & 0.08 \\
\hline $\mathrm{H}(22 \mathrm{~B})$ & $4 a$ & $0.7734(5)$ & $0.1154(3)$ & $0.3928(1)$ & 0.08 \\
\hline $\mathrm{H}(22 \mathrm{C})$ & $4 a$ & $0.7049(5)$ & $0.2107(3)$ & $0.4287(1)$ & 0.08 \\
\hline $\mathrm{H}(23 \mathrm{~A})$ & $4 a$ & $0.5542(5)$ & $0.3949(3)$ & $0.3341(1)$ & 0.08 \\
\hline $\mathrm{H}(23 \mathrm{~B})$ & $4 a$ & $0.7220(5)$ & $0.4063(3)$ & $0.3605(1)$ & 0.08 \\
\hline $\mathrm{H}(23 \mathrm{C})$ & $4 a$ & $0.5703(5)$ & $0.3791(3)$ & $0.3937(1)$ & 0.08 \\
\hline $\mathrm{H}(24 \mathrm{~A})$ & $4 a$ & $0.4222(5)$ & $0.1993(3)$ & $0.3280(1)$ & 0.08 \\
\hline $\mathrm{H}(24 \mathrm{~B})$ & $4 a$ & $0.4388(5)$ & $0.1881(3)$ & $0.3878(1)$ & 0.08 \\
\hline $\mathrm{H}(24 \mathrm{C})$ & $4 a$ & $0.5073(5)$ & $0.0927(3)$ & $0.3519(1)$ & 0.08 \\
\hline $\mathrm{H}(25 \mathrm{~A})$ & $4 a$ & $0.3826(5)$ & $0.4439(3)$ & $0.2372(1)$ & 0.08 \\
\hline $\mathrm{H}(25 \mathrm{~B})$ & $4 a$ & $0.5165(5)$ & $0.4119(3)$ & $0.1976(1)$ & 0.08 \\
\hline $\mathrm{H}(25 \mathrm{C})$ & $4 a$ & $0.5627(5)$ & $0.4659(3)$ & $0.2507(1)$ & 0.08 \\
\hline $\mathrm{H}(26 \mathrm{~A})$ & $4 a$ & $0.4622(5)$ & $0.2096(4)$ & $0.1153(1)$ & 0.08 \\
\hline $\mathrm{H}(26 \mathrm{~B})$ & $4 a$ & $0.4322(5)$ & $0.1863(4)$ & $0.1739(1)$ & 0.08 \\
\hline $\mathrm{H}(26 \mathrm{C})$ & $4 a$ & $0.5502(5)$ & $0.1086(4)$ & $0.1428(1)$ & 0.08 \\
\hline
\end{tabular}

\begin{abstract}
$\mathrm{C}_{26} \mathrm{H}_{38} \mathrm{O}_{4}$, orthorhombic, $P 2{ }_{1} 2_{1} 2_{1}$ (No. 19), $a=8.360(1) \AA$, $b=11.746(1) \AA, c=26.024(3) \AA, V=2555.5 \AA^{3}, Z=4$, $R_{\mathrm{gt}}(F)=0.067, w R(F)=0.058, T=293 \mathrm{~K}$.
\end{abstract}

\section{Source of material}

The title compound, a synthetic precursor to a structurally simplified analog of the nerve-growth stimulating mastigophorenes from liverworts [1] was prepared in enantiomerically pure form [2], using the "lactone method' [3]. 
Table 3. Atomic coordinates and displacement parameters (in $\AA^{2}$ ).

\begin{tabular}{|c|c|c|c|c|c|c|c|c|c|c|}
\hline Atom & Site & $x$ & $y$ & $z$ & $U_{11}$ & $U_{22}$ & $U_{33}$ & $U_{12}$ & $U_{13}$ & $U_{23}$ \\
\hline $\mathrm{C}(1)$ & $4 a$ & $1.0806(6)$ & $0.3453(3)$ & $0.1495(1)$ & $0.106(3)$ & $0.081(3)$ & $0.065(2)$ & $-0.036(3)$ & $0.003(2)$ & $-0.015(2)$ \\
\hline$C(2)$ & $4 a$ & $1.0446(4)$ & $0.2310(3)$ & $0.1252(1)$ & $0.054(2)$ & $0.057(2)$ & $0.043(2)$ & $-0.006(2)$ & $-0.000(2)$ & $0.001(2)$ \\
\hline $\mathrm{C}(3)$ & $4 a$ & $1.1075(4)$ & $0.2022(3)$ & $0.0771(1)$ & $0.052(2)$ & $0.066(2)$ & $0.044(2)$ & $-0.015(2)$ & $0.004(2)$ & $0.007(2)$ \\
\hline$C(4)$ & $4 a$ & $1.0695(4)$ & $0.1033(3)$ & $0.0508(1)$ & $0.052(2)$ & $0.063(2)$ & $0.037(2)$ & $-0.003(2)$ & $0.008(2)$ & $0.003(2)$ \\
\hline $\mathrm{C}(5)$ & $4 a$ & $0.9621(4)$ & $0.0293(3)$ & $0.0749(1)$ & $0.059(2)$ & $0.051(2)$ & $0.049(2)$ & $-0.008(2)$ & $0.009(2)$ & $-0.008(2)$ \\
\hline $\mathrm{O}(5)$ & $4 a$ & $0.9168(4)$ & $-0.0720(2)$ & $0.05089(9)$ & $0.097(2)$ & $0.071(2)$ & $0.073(2)$ & $-0.025(2)$ & $0.036(2)$ & $-0.024(1)$ \\
\hline$C(6)$ & $4 a$ & $0.9020(4)$ & $0.0534(3)$ & $0.1238(1)$ & $0.046(2)$ & $0.052(2)$ & $0.049(2)$ & $-0.004(2)$ & $0.010(2)$ & $-0.001(2)$ \\
\hline$O(6)$ & $4 a$ & $0.7977(3)$ & $-0.0211(2)$ & $0.14772(8)$ & $0.064(2)$ & $0.058(2)$ & $0.062(1)$ & $-0.005(1)$ & $0.023(1)$ & $-0.000(1)$ \\
\hline$C(7)$ & $4 a$ & $0.9398(4)$ & $0.1552(3)$ & $0.1491(1)$ & $0.040(2)$ & $0.058(2)$ & $0.041(2)$ & $0.006(2)$ & $-0.001(2)$ & $0.006(2)$ \\
\hline $\mathrm{C}(8)$ & $4 a$ & $0.8694(4)$ & $0.1776(3)$ & $0.2016(1)$ & $0.047(2)$ & $0.054(2)$ & $0.038(2)$ & $-0.003(2)$ & $0.002(2)$ & $-0.002(2)$ \\
\hline $\mathrm{C}(9)$ & $4 a$ & $0.7190(4)$ & $0.2286(3)$ & $0.2057(1)$ & $0.048(2)$ & $0.044(2)$ & $0.036(2)$ & $0.001(2)$ & $-0.003(2)$ & $0.004(1)$ \\
\hline $\mathrm{O}(9)$ & $4 a$ & $0.6416(3)$ & $0.2575(2)$ & $0.16042(7)$ & $0.056(1)$ & $0.064(2)$ & $0.047(1)$ & $-0.004(1)$ & $-0.006(1)$ & $0.013(1)$ \\
\hline$C(10)$ & $4 a$ & $0.6498(4)$ & $0.2525(3)$ & $0.2532(1)$ & $0.045(2)$ & $0.042(2)$ & $0.046(2)$ & $0.003(2)$ & $0.002(2)$ & $0.005(2)$ \\
\hline$O(10)$ & $4 a$ & $0.4995(3)$ & $0.3032(2)$ & $0.25502(7)$ & $0.051(1)$ & $0.052(1)$ & $0.060(1)$ & $0.007(1)$ & $0.006(1)$ & $0.007(1)$ \\
\hline $\mathrm{C}(11)$ & $4 a$ & $0.7263(4)$ & $0.2185(3)$ & $0.2987(1)$ & $0.055(2)$ & $0.046(2)$ & $0.039(2)$ & $0.005(2)$ & $0.003(2)$ & $-0.006(2)$ \\
\hline $\mathrm{C}(12)$ & $4 a$ & $0.8755(4)$ & $0.1661(3)$ & $0.2939(1)$ & $0.052(2)$ & $0.062(2)$ & $0.042(2)$ & $0.006(2)$ & $-0.004(2)$ & $0.002(2)$ \\
\hline$C(13)$ & $4 a$ & $0.9515(4)$ & $0.1474(3)$ & $0.2466(1)$ & $0.049(2)$ & $0.062(2)$ & $0.045(2)$ & $0.010(2)$ & $-0.006(2)$ & $-0.013(2)$ \\
\hline $\mathrm{C}(14)$ & $4 a$ & $1.1162(4)$ & $0.0947(3)$ & $0.2450(1)$ & $0.062(2)$ & $0.094(3)$ & $0.053(2)$ & $0.027(2)$ & $-0.010(2)$ & $-0.004(2)$ \\
\hline$C(15)$ & $4 a$ & $1.1407(5)$ & $0.0773(3)$ & $-0.0027(1)$ & $0.066(3)$ & $0.075(3)$ & $0.043(2)$ & $0.001(2)$ & $0.017(2)$ & $0.003(2)$ \\
\hline$C(16)$ & $4 a$ & $1.2518(6)$ & $0.1716(4)$ & $-0.0205(1)$ & $0.117(4)$ & $0.158(5)$ & $0.059(2)$ & $-0.065(4)$ & $0.040(3)$ & $-0.023(3)$ \\
\hline $\mathrm{C}(17)$ & $4 a$ & $1.2350(7)$ & $-0.0345(4)$ & $-0.0019(1)$ & $0.123(4)$ & $0.160(5)$ & $0.072(3)$ & $0.049(5)$ & $0.047(3)$ & $0.019(3)$ \\
\hline $\mathrm{C}(18)$ & $4 a$ & $1.0083(5)$ & $0.0678(4)$ & $-0.0435(1)$ & $0.098(3)$ & $0.095(3)$ & $0.047(2)$ & $-0.003(3)$ & $0.000(2)$ & $-0.014(2)$ \\
\hline $\mathrm{C}(19)$ & $4 a$ & $0.7500(6)$ & $-0.0823(5)$ & $0.0406(2)$ & $0.121(5)$ & $0.154(5)$ & $0.080(3)$ & $-0.075(4)$ & $0.030(3)$ & $-0.044(3)$ \\
\hline $\mathrm{C}(20)$ & $4 a$ & $0.8700(6)$ & $-0.1194(4)$ & $0.1690(2)$ & $0.098(4)$ & $0.085(3)$ & $0.090(3)$ & $-0.014(3)$ & $0.025(3)$ & $0.020(3)$ \\
\hline $\mathrm{C}(21)$ & $4 a$ & $0.6482(4)$ & $0.2386(3)$ & $0.3525(1)$ & $0.070(2)$ & $0.057(2)$ & $0.037(2)$ & $0.005(2)$ & $0.010(2)$ & $0.004(2)$ \\
\hline$C(22)$ & $4 a$ & $0.7558(5)$ & $0.1957(3)$ & $0.3964(1)$ & $0.098(3)$ & $0.084(3)$ & $0.038(2)$ & $0.006(3)$ & $0.010(2)$ & $0.001(2)$ \\
\hline $\mathrm{C}(23)$ & $4 a$ & $0.6216(5)$ & $0.3668(3)$ & $0.3612(1)$ & $0.082(3)$ & $0.064(3)$ & $0.060(2)$ & $0.003(2)$ & $0.016(2)$ & $-0.015(2)$ \\
\hline$C(24)$ & $4 a$ & $0.4896(5)$ & $0.1731(3)$ & $0.3554(1)$ & $0.087(3)$ & $0.070(3)$ & $0.060(2)$ & $-0.001(3)$ & $0.028(2)$ & $-0.002(2)$ \\
\hline $\mathrm{C}(25)$ & $4 a$ & $0.4897(5)$ & $0.4157(3)$ & $0.2334(1)$ & $0.063(2)$ & $0.053(2)$ & $0.082(2)$ & $0.014(2)$ & $-0.004(2)$ & $0.010(2)$ \\
\hline$C(26)$ & $4 a$ & $0.5105(5)$ & $0.1848(4)$ & $0.1469(1)$ & $0.068(3)$ & $0.091(3)$ & $0.061(2)$ & $-0.007(3)$ & $-0.017(2)$ & $0.009(2)$ \\
\hline
\end{tabular}

\section{References}

1. Fukuyama, Y.; Asakawa, Y.: Novel Neurotorphic Isocuparane-type Sesquiterpene Dimers, Mastigophorenes A, B, C and D, Isolated from the Liverwort Mastigophora dicladus. J. Chem. Soc. Perkin Trans. I (1991) $2737-2741$
2. Bringmann, G.; Pabst, T.; Busemann, S.; Peters, K.; Peters, E.-M. Atropo-Enantioselective Synthesis of a Simplified Analog of Mastigophorenes A und B. Tetrahedron 54 (1998) 1425-1438.

3. Bringmann, G.; Breuning, M.; Tasler, S.: The Lactone Concept: An Efficient Pathway to Axially Chiral Natural Products and Useful Reagents. Synthesis (1999) 525-558.

4. Sheldrick, G. M.: Program Package SHELXTL-Plus. Release 4.1, Siemens Analytical X-Ray Instruments Inc., Madison (WI 53719), USA 1990. 